\title{
ESTRATÉGIAS EDUCATIVAS NO TRANSPLANTE PEDIÁTRICO DE ÓRGÃOS SÓLIDOS: REVISÃO INTEGRATIVA DA LITERATURA
}

\author{
Educational strategies in pediatric solid organ transplantation: \\ an integrative literature review
}

\author{
Paloma Peroni Contiero', Cristina Maria Galvão², Karina Dal Sasso Mendes²
}

\begin{abstract}
RESUMO
Objetivo: Analisar evidências disponíveis na literatura sobre estratégias utilizadas para o ensino de candidatos e receptores pediátricos de transplante de órgãos sólidos. Método: Revisão integrativa da literatura com seleção de artigos nas bases de dados PubMed, EMBASE, CINAHL e LILACS. Resultado: Identificou-se o uso de plataformas online e eletrônicas, gadgets que auxiliam na adesão ao tratamento pós-transplante, acampamentos familiares, materiais impressos e vídeos, como as estratégias utilizadas para o ensino no transplante de pacientes pediátricos. Conclusão: Há escassez de estudos na literatura nacional e internacional quanto ao ensino de pacientes pediátricos no contexto do transplante, identificando-se a necessidade de novas investigações, tendo em vista a importância do ensino para a continuidade do cuidado e da melhor qualidade de vida do paciente pediátrico.
\end{abstract}

Descritores: Transplante de Órgãos; Pediatria; Materiais de Ensino; Educação; Revisão

\section{Instituição:}

1. Curso de Bacharelado e Licenciatura em Enfermagem da Escola de Enfermagem de Ribeirão Preto da Universidade de São Paulo, Ribeirão Preto, São Paulo, Brasil.

2. Departamento de Enfermagem Geral e Especializada da Escola de Enfermagem de Ribeirão Preto da Universidade de São Paulo, Ribeirão Preto, São Paulo, Brasil.

\section{Correspondência:}

Karina Dal Sasso Mendes

E-mail: dalsasso@eerp.usp.br

https://doi.org/10.53855/bjt.v24i2.014

Recebido em: 14/01/2021
Aceito em: 27/042021

\section{INTRODUÇÃO}

Com o sucesso do transplante, novos desafios são enfrentados por crianças e adolescentes receptores de órgãos. O transplante em si é capaz de substituir a doença em fase terminal por um estado de doença crônica manejável e sustentável, podendo gerar profundas consequências clínicas e psicossociais no indivíduo. ${ }^{1}$ Assim, lidar com candidatos e receptores de transplante pediátrico e seus familiares configura-se em um desafio para o enfermeiro no transplante.

Segundo dados do Registro Brasileiro de Transplantes (RBT), da Associação Brasileira de Transplante de Órgãos (ABTO), o número de transplantes pediátricos realizado no Brasil no ano de 2019 foi de 584 crianças, ou seja, $53 \%$ dos pacientes pediátricos que estavam 
em lista de espera (1.093). ${ }^{2}$ Ao comparar esses dados com anos anteriores, observa-se aumento no número de transplantes pediátricos a cada ano.

Crianças e seus familiares que enfrentam o processo de transplante lidam com múltiplas e únicas demandas. Essas demandas envolvem o nível de desenvolvimento da criança, a dinâmica familiar e o fato de que muitas crianças são muito jovens e têm pouca ou nenhuma participação ou controle sobre seus próprios cuidados. Diferentes problemas de saúde são encontrados, dependendo do tipo de transplante que a criança necessita. $^{3}$

A falência de órgãos em fase terminal difere de outras doenças da infância com risco de vida, onde o transplante oferece chance de sobrevida e reabilitação em longo prazo. No entanto, crianças com falência de órgãos estão em risco de ter problemas físicos e emocionais semelhantes às crianças com outras doenças crônicas e potencialmente fatais. Antes do transplante, as crianças incorrem em repetidas hospitalizações, que podem envolver separação de membros da família, da escola, dos amigos e do ambiente familiar. Em vez disso, um novo ambiente, o hospital, com número desconcertante de novos profissionais de saúde e a ameaça adicional de procedimentos médicos dolorosos tornam-se necessários. Durante todo o processo de transplante, a criança é influenciada por experiências anteriores com os serviços de saúde. A percepção das experiências das crianças, tanto no pré quanto no pós-transplante poderá ser afetada por seu desenvolvimento cognitivo e etário. ${ }^{3}$

Segundo a literatura, é possível que crianças receptoras de órgãos possam apresentar distúrbios emocionais, comportamentais e sociais, sendo imaturidade, ansiedade relacionada à perda e separação, inibição, alteração na imagem corporal, retraimento, somatização, regressão, sintomas depressivos, problemas de atenção e de comportamento, baixa autoestima e baixo escore no quociente de inteligência (QI), alguns dos sintomas mais comumente encontrados em crianças transplantadas ou que ainda aguardam na fila de espera. ${ }^{4,5} \mathrm{~A}$ experiência da doença crônica ocasiona efeitos no desenvolvimento da criança devido ao seu estado físico e emocional afetados. ${ }^{4,6}$

No transplante infantil é evidente o impacto psicológico não somente na criança, mas também em seus familiares. A condição de saúde do paciente demanda maiores esforços dos demais membros da família ao tentarem ajustar-se ao processo da doença, gerando sentimentos de ansiedade, depressão, culpa e frustração nos pais, enquanto irmãos podem sentir-se excluídos do meio familiar devido à menor atenção recebida para si, demonstrando problemas comportamentais. ${ }^{6-7}$ Apesar da possibilidade de efeitos negativos para a família, o manejo e suporte familiar possui grande importância no cuidado da criança/adolescente, assim como em seu prognóstico e enfrentamento do próprio paciente frente à doença. ${ }^{4,6-9}$

Com os diversos efeitos negativos que podem ser gerados tanto no paciente pediátrico quanto em seus familiares durante o tratamento é primordial a presença de uma equipe de saúde bem preparada para proporcionar apoio às necessidades físicas e emocionais de ambos. Tal apoio envolve cuidados básicos ao paciente, o envolvimento emocional com estabelecimento de vínculo profissional-paciente e ações educativas, capazes de facilitar o processo de transplante..$^{10-11}$

Dificuldades já encontradas no transplante adulto podem ser exacerbadas na população pediátrica, principalmente no âmbito emocional. A falta de maturidade da criança e do adolescente pode afetar sua compreensão referente ao transplante, dificultar a aceitação do tratamento e impactar na qualidade de vida. O apoio familiar é essencial nesse contexto. A compreensão dos pais sobre o processo de transplante pode se refletir na criança de forma positiva ou negativa, a depender de como a família lida com a situação. Assim, torna-se de suma importância uma equipe de saúde preparada para fornecer informações pertinentes e necessárias tanto à família quanto à criança.

Frente ao exposto, questiona-se como tem sido a abordagem de ensino referente ao transplante para candidatos e receptores de órgãos sólidos pediátricos, como acontece esse ensino, quem fornece esse conhecimento para crianças e como tal ensino pode ajudar na aceitação da criança e da família ao tratamento. Tais indagações motivaram a realização do presente estudo. Portanto, o objetivo deste estudo é analisar as evidências disponíveis na literatura sobre as estratégias utilizadas para o ensino de candidatos e receptores pediátricos de transplante de órgãos sólidos.

\section{MÉTODOS}

A revisão integrativa $(\mathrm{RI})$ foi o método adotado para a condução do presente estudo, no qual foram adotados os seguintes passos: elaboração da questão de pesquisa, amostragem ou busca na literatura de estudos primários, extração de dados, avaliação dos estudos incluídos na revisão, análise e síntese dos resultados e apresentação da revisão integrativa. ${ }^{12}$

A questão norteadora para condução da RI foi pautada na estratégia PICO, acrônimo de Paciente ou problema 
(candidatos e receptores pediátricos), Intervenção (estratégias de ensino utilizadas), Controle ou comparação (não se aplica neste estudo) e Outcome ou Desfecho (processo de transplante de órgãos sólidos), que consistiu em: Quais são as evidências disponíveis na literatura sobre as estratégias utilizadas para o ensino de candidatos e receptores pediátricos submetidos ao transplante de órgãos sólidos?

Para a busca dos estudos primários foi utilizada a internet para acessar as bases de dados: Literatura LatinoAmericana e do Caribe em Ciências da Saúde (LILACS), National Library of Medicine and the National Institutes of Health (PubMed), Cumulative Index to Nursing and Allied Health Literature (CINAHL) e EMBASE. Para tanto, foram utilizados descritores controlados da Medical Subject Headings (MeSH), na List of Headings (CINAHL), no Emtree (EMBASE) e os Descritores em Ciências da Saúde (DeCS), delimitados de acordo com cada base de dados.

Os critérios para inclusão dos estudos selecionados foram: estudos primários que abordavam as estratégias de ensino utilizadas em crianças sobre o processo de transplante; nos idiomas português, inglês e espanhol. Foram excluídos editoriais e cartas respostas.

Para a estratégia de busca nas bases de dados selecionadas foram realizadas combinações entre os descritores, com auxílio dos operadores booleanos (OR e AND) e de acordo com o acrônimo PICO, conforme demonstrado a seguir: ("Waiting Lists" OR "Waiting List" OR "Transplant Recipients" OR "Transplant Recipient" OR "Pediatrics" OR "Child" OR "Children" OR "Child Care" OR "Puericulture" OR "Child Day Care") AND ("Patient Education as Topic" OR "Patient Education" OR "Education of Patients" OR "Teaching" OR "Training Techniques" OR "Training Technique" OR "Training Technics" OR "Training Technic" OR "Pedagogy" OR "Pedagogies" OR "Teaching Methods" OR "Teaching Method" OR "Academic Training" OR "Training Activities" OR "Training Activity" OR "Educational Technics" OR "Educational Technic" OR "Educational Techniques" OR "Educational Technique" OR "Teaching Materials" OR "Teaching Material") AND ("Organ Transplantation" OR "Organ Transplantations" OR "Organ Grafting" OR "Organ Graftings")

Os resultados da combinação entre os descritores nas bases de dados selecionadas foram exportados para o gerenciador de referências bibliográficas EndNote ${ }^{\circledR}$ (versão 2017), no qual foi realizado o gerenciamento das referências para manejo das duplicatas. Após exclusão dos duplicados, os resultados foram exportados para o aplicativo online Rayyan, ${ }^{13}$ utilizado para a seleção de artigos para revisões da literatura, o qual proporciona seleção semiautomática dos artigos e maior rigor para o método.

Para a primeira seleção dos estudos foi realizada leitura de título e resumo. A seguir, realizou-se a leitura na íntegra dos artigos. Os estudos que responderam à questão norteadora e aos critérios de seleção foram selecionados para compor a amostra da revisão integrativa.

Para extração dos dados foi utilizado roteiro adaptado da literatura ${ }^{14}$ e foi construído quadro com a síntese de cada estudo selecionado, contemplando dados de identificação do artigo, instituição sede, tipo de revista, características metodológicas, entre outros.

Para a identificação do delineamento dos estudos primários, foi adotada a nomenclatura utilizada pelos próprios autores das pesquisas. Quando não houve a identificação do tipo de estudo, a análise do delineamento foi fundamentada nos conceitos da literatura especializada. ${ }^{15}$

A análise e a síntese dos dados foram realizadas na forma descritiva, possibilitando ao leitor a síntese de cada estudo incluído na revisão integrativa, bem como a identificação de lacunas do conhecimento para o desenvolvimento de futuras investigações nesta temática.

\section{RESULTADOS}

O fluxograma PRISMA demonstra o caminho percorrido para a seleção final dos estudos. (Figura 1).

Dos sete artigos selecionados na amostra final, todos foram publicados em inglês, com predominância de autoria de profissionais da Medicina (três), dois estudos de autoria principal de profissionais da Psicologia e apenas um estudo com autoria de profissional da Enfermagem e de Ciências Sociais. Tratando-se do local do estudo, houve predominância de países como Canadá e Estados Unidos da América (EUA), com apenas um estudo sendo realizado na Suíça, o que demonstrou escassez de trabalhos relacionados à temática produzidos no Brasil. A Tabela 1 a seguir, apresenta a caracterização dos estudos incluídos nessa revisão segundo autores, ano, local do estudo, delineamento, autoria e periódico.

Observou-se que a maioria dos estudos incluídos foram realizados em hospitais-escola e publicados em revistas da área médica com enfoque no transplante de órgãos pediátricos. A Tabela 2 apresenta a síntese dos estudos primários segundo objetivo, método, resultados e conclusão. 
Figura 1 - Fluxograma de identificação, seleção e inclusão dos estudos da RI

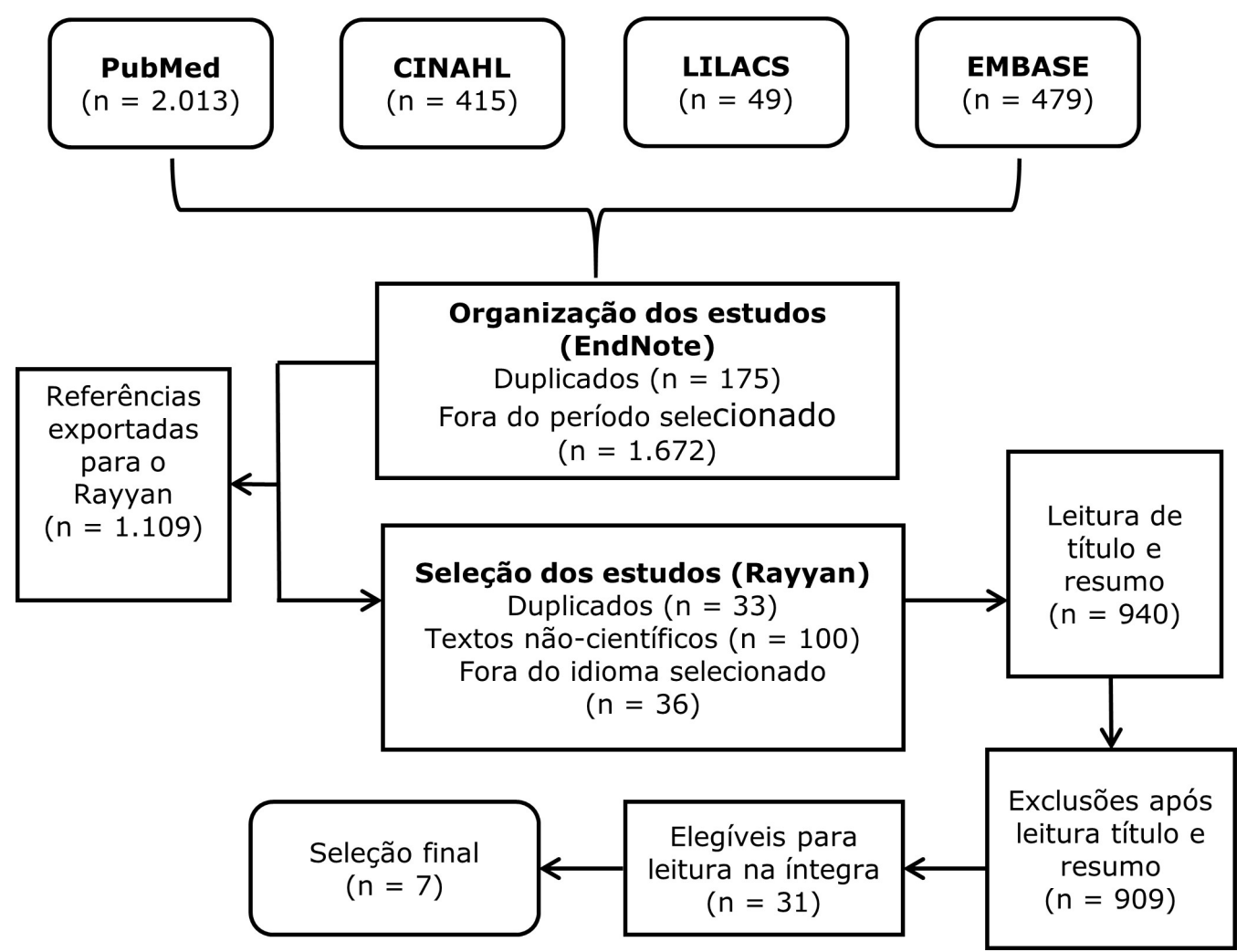

Tabela 1 - Características dos estudos primários segundo autores, ano, local do estudo, delineamento, autoria e periódico.

\begin{tabular}{|c|c|c|c|c|}
\hline Autores/Ano & Local do Estudo & Delineamento & Autoria & Área do Periódico \\
\hline (E1) McLin, V. et al $(2013)^{24}$ & Genebra, Suíça & Estudo Metodológico & Médica & Tecnologia em Saúde \\
\hline $\begin{array}{l}\text { (E2) Kullgren, KA. et al } \\
(2015)^{29}\end{array}$ & St. Louis, EUA & Ensaio Clínico Randomizado & Psicologia & $\begin{array}{l}\text { Médico (Transplante de } \\
\text { Órgãos Infantil) }\end{array}$ \\
\hline (E3) Korus, M. et al $(2015)^{18}$ & Toronto, Canadá & $\begin{array}{l}\text { Teste de Usabilidade } \\
\text { Qualitativa }\end{array}$ & Enfermagem & $\begin{array}{l}\text { Médico (Transplante de } \\
\text { Órgãos Infantil) }\end{array}$ \\
\hline $\begin{array}{l}\text { (E4) Shellmer, DA. et al } \\
(2016)^{27}\end{array}$ & $\begin{array}{l}\text { Pittsburgh, } \\
\text { EUA }\end{array}$ & Teste de Usabilidade & Psicologia & $\begin{array}{l}\text { Médico (Transplante de } \\
\text { Órgãos Infantil) }\end{array}$ \\
\hline $\begin{array}{l}\text { (E5) Nicholas, DB. et al } \\
(2016)^{30}\end{array}$ & Edmonton, Canadá & $\begin{array}{l}\text { Estudo misto, quase- } \\
\text { experimental, pré e pós } \\
\text { teste }\end{array}$ & Serviço Social & Ciências Sociais na Saúde \\
\hline $\begin{array}{l}\text { (E6) Coughlin, CC. et al } \\
(2017)^{31}\end{array}$ & St. Louis, EUA & $\begin{array}{l}\text { Estudo Prospectivo Não- } \\
\text { Randomizado }\end{array}$ & Médica & $\begin{array}{l}\text { Médica (Transplante de } \\
\text { Órgãos Infantil) }\end{array}$ \\
\hline $\begin{array}{l}\text { (E7) Foster, BJ. et al } \\
(2018)^{28}\end{array}$ & Montreal, Canada & $\begin{array}{l}\text { Ensaio Clínico } \\
\text { Randomizado }\end{array}$ & Médica & $\begin{array}{l}\text { Multiprofissional } \\
\text { (Nefrologia) }\end{array}$ \\
\hline
\end{tabular}


Tabela 2 - Síntese dos estudos primários segundo objetivo, método e principais resultados

$\begin{array}{llll}\text { Estudo Objetivo } & \text { Método Principais Resultados }\end{array}$

$\mathrm{E}^{24} \quad$ Construir plataforma de ensino para o paciente pediátrico e familiares.

\section{$E 2^{29}$}

Determinar a eficácia do produto comercial "garrafa d'agua interativa" na melhora da ingestão de líquidos recomendada no pós-transplante de pacientes pediátricos e avaliar melhora da função renal, comparado ao cuidado padrão.

\section{E31 ${ }^{8}$ Desenvolver, testar e avaliar} a usabilidade, eficiência e aceitação dos três primeiros módulos de "Teens Taking Charge: Managing My Transplant Online", ferramenta online composta por 12 módulos voltada para o ensino sobre o transplante de órgãos sólidos para adolescentes.

$\mathrm{E}^{27}$ Descrever o processo de desenvolvimento e teste do aplicativo "Teen Pocket PATH" (TPP), assim como avaliar a aceitação, usabilidade e satisfação dos participantes, e as características finais do aplicativo.

\section{E5 $^{30}$ Explorar o impacto de} acampamento familiar como intervenção educativa e de apoio social para receptores de transplante cardíaco pediátricos e seus familiares.

\section{E6 $^{31}$ Avaliar o conhecimento} sobre o câncer de pele, a proteção solar, a autoeficácia e as barreiras quanto à fotoproteção em pacientes pediátricos receptores de órgãos e seus familiares.

E7 28 Testar a eficácia da intervenção - "TAKE-IT" (Teen Adherence in Kidney transplant, Effectiveness of Intervention Trial) - assim como caracterizar e comparar a adesão ao tratamento medicamentoso entre os grupos (GI e GC).
A equipe multiprofissional da saúde e da computação foram recrutadas para a construção da plataforma "KidsETransplant", com informações gerais acerca do transplante de fígado; fórum para discussão entre pacientes e familiares; e aplicativo para acessar o prontuário eletrônico.

32 pacientes (7 a 19 anos) foram divididos entre grupos GC* e GI**. O GI recebeu a garrafa d'água interativa e foi instruído quanto ao uso da mesma. Ao receber uma meta diária de ingestão de líquidos, todos os participantes ( $G$ l e GC) deveriam manter anotações quanto à quantidade de líquidos ingeridos por dia em um diário.

A plataforma obteve feedback positivo das crianças. Foram planejadas melhorias com a inclusão de caixas de texto associadas a determinados objetivos na sala virtual das crianças, e a adição de perguntas desafiadoras nos jogos propostos.

Quase $90 \%$ dos GI atingiu ou excedeu as taxas de ingestão de líquidos diária em comparação a $40 \%$ do GC. Os níveis sanguíneos de nitrogênio uréico aumentaram no GC el diminuíram no GI. O nível de sódio diminuiu no GC. Houve aumento nos níveis de creatinina em ambos os grupos. $50 \%$ dos participantes se sentiram confortáveis ao usar a garrafa d'água em público e a mesma ajudou na ingestão hídrica.

Pacientes transplantados entre 12 e 18 anos de idade testaram os módulos "Medicamentos e Vacinas", "Alimentação após o Transplante" e "A vida após o Transplante de Órgão" do website criado com informações coletadas de estudo qualitativo e revisão da literatura. Após teste, os participantes forneceram sugestões para melhoria no desenvolvimento do website.

Sete adolescentes (11 a 18 anos), juntamente com seus cuidadores, testaram o aplicativo TPP. Foram realizadas 3 sessões para coleta de informações quanto ao tratamento medicamentoso, e o desenvolvimento inicial do protótipo. Após o desenvolvimento do aplicativo, foi realizado teste piloto durante 6 semanas.

A navegação do Website melhorou entre a primeira e a terceira interações. Os erros de navegação diminuíram de $20 \%$ para $6 \%$ (primeira e segunda interações), e nenhum erro na terceira. O layout foi classificado como intuitivo. As informações foram consideradas de fácil entendimento e relevantes. Os participantes afirmaram que usariam a plataforma, recomendando-a para outros adolescentes transplantados.

Oaplicativofoidesenvolvidocom diversoscomponentes que ajudaram no cuidado pós-transplante, fornecendo informações de alta hospitalar e promovendo maior suporte para adesão ao tratamento medicamentoso. O aplicativo foi considerado de fácil uso e efetivo ao manter a adesão medicamentosa dos adolescentes.

O acampamento foi criado para oferecer apoio e atividades educativas aos pacientes e familiares, por meio de atividades recreativas e educacionais. A avaliação do acampamento foi realizada pela observação, entrevistas, medidas padronizadas antes e após o acampamento e avaliação da satisfação dos participantes.

Receptores pediátricos (10 a 17 anos) e familiares compuseram Gl*. O GC** foi composto por pacientes da clínica de dermatologia, submetidos ao cuidado usual. Realizada avaliação inicial com caracterização, histórico médico e conhecimento sobre o câncer de pele. Realizada intervenção educacional com a utilização de informação oral, vídeos e materiais impressos. O pós-teste sobre o conhecimento foi reaplicado após 1 semana, 3 e 6 meses.

169 pacientes (11 a 24 anos), divididos entre GC* e Gl**, situados em oito centros de transplante renal pediátrico do Canadá e dos EUA participaram da intervenção "TAKE-IT" que possui componentes educacionais, organizacionais e comportamentais para atingir barreiras comuns na adesão ao tratamento medicamentoso. A adesão ao tratamento foi identificada pelo controle da ingestão do medicamento, avaliação do enxerto e eventos adversos.
49 pessoas (incluindo crianças, adolescentes, familiares e profissionais de saúde) realizaram a avaliação. Pacientes alegaram ganho de conhecimento acerca do transplante cardíaco após o acampamento. Familiares aprenderam sobre as experiências do transplante cardíaco pediátrico, riscos do tratamento, diferenças entre as doenças cardíacas e os desafios do enfrentamento a esta nova condição de saúde.

O conhecimento aumentou significativamente após a intervenção educacional realizada com GI (pacientes e familiares). Os comportamentos relacionados à proteção solar se tornaram mais frequentes após a intervenção. A auto eficácia se mostrou maior no Gl do que no GC, porém os familiares não apresentaram diferenças significativas após a intervenção educativa.

Observou-se que a adesão medicamentosa do GI melhorou consideravelmente logo após o primeiro período da intervenção e se manteve até o fim. Com relação aos níveis do imunossupressor, não houve diferença entre os grupos, assim como o autorrelato dos participantes. O índice de rejeição foi menor no Gl. 


\section{DISCUSSÃO}

A busca na literatura evidenciou que crianças com necessidade de transplante podem apresentar déficit cognitivo durante seu desenvolvimento, por consequência do rápido crescimento neurológico, dos efeitos adversos do tratamento medicamentoso e dos aspectos da própria doença, que diminuem a frequência em atividades escolares. Tais fatores geram dificuldades no desempenho escolar da criança, afetando também a vida social, uma vez que, nessa idade, a ausência na escola e em atividades recreacionais podem acarretar maior risco de isolamento social. ${ }^{1,6}$ Porém, foi demostrado que, após o transplante, o déficit cognitivo tende a retornar aos níveis mais próximos dos padrões de normalidade esperado para a faixa etária da criança. ${ }^{1}$

Muitas crianças que enfrentam o transplante como tratamento apresentam comportamento de baixa tolerância à frustração, teimosia, sentimento de insegurança e desamparo, assim como dificuldade de relacionamento e comportamentos regressivos, ou seja, comportamentos da infância que se perpetuam para a maior idade. Ao atingir a adolescência, manifestam maior necessidade de independência, autonomia, conhecimento e desenvolvimento de atitudes e habilidades que ajudam no auto manejo da doença. ${ }^{6-7}$ Porém, a relação de dependência entre pais e filhos, em condições de saúde precárias, ocorre em níveis além do esperado e relaciona-se à dependência inerente ao acompanhamento médico e medicamentoso. ${ }^{4,6} \mathrm{~A}$ literatura apresentou dados que indicam a relação direta entre a forma como a família trata a criança e a doença e a maneira como a criança se desenvolve. Como exemplo, a literatura aponta que crianças que demonstram capacidade de agir de maneira autônoma e independente são frutos de mães que aceitaram a doença e o tratamento de forma natural, não alterando o cuidado da criança devido às suas limitações. ${ }^{4}$

É importante salientar a adolescência como uma das fases de desenvolvimento infantil com maior nível de estresse para o indivíduo em condições saudáveis de vida, assim como, para aqueles que apresentam algum estado de doença crônica ou terminal. ${ }^{16}$ Trata-se de fase complicada para pacientes em processo de transplante, já que é esperado do adolescente maior responsabilidade quanto ao seu auto manejo. Além disso, é normal que os pais permitam maior independência aos seus filhos nessa fase da vida. Apesar disso, alguns jovens ainda não possuem maturidade suficiente para praticar o autocuidado adequado no pós-transplante, consequência do possível atraso em seu desenvolvimento durante o processo de doença. Por tais motivos, a adolescência é conhecida por ser a fase de maior vulnerabilidade para pacientes pediátricos, sendo comum a não adesão ao tratamento medicamentoso, acarretando complicações e risco de perda do enxerto. ${ }^{1,17}$

Estudo realizado para explorar as necessidades de informação de adolescentes, após transplante renal, evidenciou quatro fatores estressantes apontados pelos jovens durante o processo do transplante: (1) imagem corporal; (2) desejo de ser normal; (3) dor e desconforto e (4) quebra na comunicação entre paciente e equipe de saúde. ${ }^{16}$ A forma encontrada pelos adolescentes neste estudo para enfrentar tais barreiras foi obter conhecimento acerca da doença e do tratamento, além de garantir apoio emocional de familiares e amigos. Esses achados corroboram com evidências da literatura, as quais mostraram que compreender melhor a doença e seu tratamento, somado ao suporte de família e amigos, é a maneira que pacientes pediátricos encontram para enfrentar sua situação de saúde, proporcionando maior adesão ao tratamento e melhoria no auto manejo da doença. ${ }^{6-7}$

A literatura também traz evidências sobre a importância da equipe de saúde dosar a atenção do tratamento entre o paciente pediátrico e seus familiares, não direcionando as informações e perguntas apenas para os cuidadores da criança/adolescente, mas sim para o próprio paciente, focando em suas necessidades de informação de forma individualizada e, também, permitindo que o paciente possa vir a vivenciar maiores oportunidades de aquisição de sua autonomia e independência. ${ }^{6-7}$

Os dados coletados no estudo qualitativo citado acima foram utilizados para desenvolver o programa online intitulado "Teens Taking Charge: Managing My Transplant Online" apresentado como estudo selecionado nesta revisão. ${ }^{18}$ Tendo como base as necessidades de informação demonstradas pelos jovens submetidos ao transplante renal, ${ }^{16}$ o programa online foi desenvolvido com base em informações referentes ao cuidado após o transplante, necessário para o autocuidado de pacientes pediátricos. $^{18}$ Tal programa consistiu em 12 módulos que abordaram assuntos pertinentes ao auto manejo do paciente. Para o estudo em questão, foram desenvolvidos três módulos do programa ("Medicamentos e Vacinas"; "Alimentação após o Transplante"; e "A vida após o Transplante de Órgão"). A partir do teste de usabilidade dos módulos pelos jovens transplantados, entre 12 e 18 anos de idade, foram destacados resultados positivos com informações de forma compreensível, detalhada e com linguagem de fácil acesso através do programa online.

Apesar dessa plataforma ser promissora para o futuro do ensino de candidatos e receptores pediátricos, é interessante ressaltar que, em outro estudo,16 os 
jovens também trouxeram, além da necessidade de uma plataforma online que pudesse fornecer informações fidedignas de maneira rápida, segura e de fácil acesso para pacientes e seus familiares, a importância da educação individualizada junto ao profissional de saúde. A literatura sugere que, para a educação efetiva e benéfica ao paciente pediátrico e seus familiares, é necessário que exista vínculo entre os profissionais, os pacientes e familiares, capaz de permitir a confiança entre todos os envolvidos, facilitando o papel educativo do profissional, contribuindo para o desenvolvimento de habilidades para o auto manejo, para a adesão ao tratamento e melhoria na qualidade de vidal. ${ }^{11}$

Para que a educação individualizada seja realizada de forma efetiva, o profissional deve avaliar o nível cognitivo e de alfabetização da criança, utilizar materiais de apoio, linguagem e postura, de modo a facilitar a compreensão das informações. .119-20 $^{1,2}$ Alfabetização ou literácia em saúde é a habilidade do indivíduo em compreender e aplicar as informações que são fornecidas pelos profissionais de saúde quanto à doença e ao tratamento. Assim, entender que crianças possuem maneira particular de entender o mundo à sua volta, necessitando de estratégias específicas e linguagem adequada para que as informações possam ser apreendidas de maneira significativa é de grande valia. Seus pais, como principais cuidadores, devem compreender as informações recebidas, a fim de propiciar o manejo da doença. ${ }^{21}$

O enfermeiro, como parte integrante da equipe de saúde, está presente em todo o processo do transplante desde a avaliação do potencial receptor até o período de cuidados pós-transplante. Por ser o profissional que possui maior contato com pacientes e familiares, o estabelecimento de vínculo, planejamento de ações e ensino são parte fundamental de sua prática. ${ }^{10}$ Esses profissionais são capacitados para a identificação de fatores que podem influenciar no bem-estar de pacientes que se encontram no processo de transplante, oferecendo suporte emocional e informações. ${ }^{22}$ Estudos enfatizam que a falta de informação pode aumentar sentimentos de ansiedade e medo no paciente pediátrico e seus familiares, sendo importante o planejamento de estratégias de ensino-aprendizagem sobre o procedimento cirúrgico e as repercussões que a criança irá enfrentar, a fim de auxiliar na diminuição de sentimentos negativos que interfiram na recuperação e cuidado pós-operatório. ${ }^{19,23}$

Nessa direção, estudo trouxe o desenvolvimento da plataforma denominada "KidsETransplant", ${ }^{24}$ com enfoque dado para crianças receptoras de fígado e seus familiares. Tal plataforma foi desenvolvida pela necessidade de maximizar as chances de sucesso do transplante pediátrico, permitindo o ensino dos pacientes e seus familiares por meio de website contendo informações gerais sobre doenças do fígado, tratamento, manejo dos cuidados, entre outros tópicos. Além disso, também apresenta fórum interativo para discussão entre pacientes e familiares visando troca de informações e experiências. $\mathrm{O}$ aplicativo interativo permite acesso ao prontuário médico do paciente, contendo informações de resultados laboratoriais, tratamento medicamentoso, datas de consultas médicas, entre outros.

Tecnologias como websites, aplicativos móveis e até mesmo o uso de gadgets vêm ganhando espaço e auxiliando de maneira positiva em avanços na área da saúde. Uma variedade de jogos educativos (como vídeo games) vem auxiliando na promoção do cuidado, contribuindo para a conquista de determinados objetivos e o desenvolvimento de habilidades específicas para o auto manejo do paciente. ${ }^{25}$ Plataformas educativas em websites, capazes de fornecer informações ao indivíduo de maneira interativa, e por vezes individualizada, permite o avanço no tratamento, e até mesmo mudanças de comportamento capazes de auxiliar na adesão ao tratamento. ${ }^{26}$

Corroborando com esses achados, outra pesquisa mostrou as etapas de desenvolvimento do aplicativo móvel chamado "Teen Pocket PATH" (TPP), ${ }^{27}$ criado para auxiliar na melhoria da adesão medicamentosa de adolescentes transplantados. Neste estudo, a equipe de pesquisadores desenvolveu o TPP com base em dados coletados em entrevista com jovens entre 11 e 18 anos de idade e seus principais cuidadores, acerca do manejo medicamentoso e das barreiras encontradas para a manutenção do tratamento. O protótipo do aplicativo foi testado pelos adolescentes e seus cuidadores. A premissa básica do TPP era fornecer informações sobre os medicamentos utilizados pelo adolescente e auxiliar no controle do seu uso, de acordo com a prescrição fornecida pela equipe médica, incluindo informações para os cuidadores requeridos pelo paciente. O TPP permitiu o aumento da independência e autonomia do paciente pediátrico manteve certo nível de supervisão dos cuidadores, de maneira a não interferir no processo de desenvolvimento dos filhos. Os resultados mostraram que o aplicativo era de fácil usabilidade e auxiliou na melhoria da comunicação entre os pacientes e seus familiares, uma vez que houve menor necessidade de perguntar sobre a tomada dos medicamentos.

No cenário tecnológico, os estudos analisados trouxeram a utilização de gadgets para auxiliar em aspectos do cuidado pós-transplante no que se refere à adesão medicamentosa ${ }^{28}$ e à ingestão de líquidos 
na recuperação do transplante renal. ${ }^{29} \mathrm{O}$ ensaio clínico randomizado 28 demostrou, além da utilização da caixa eletrônica de comprimidos para monitoramento do tratamento medicamentoso, o impacto de sessões educativas, organizacionais e comportamentais junto aos jovens participantes do estudo (entre 11 e 24 anos de idade), em intervalos regulares de tempo, visando aumentar a adesão medicamentosa desses adolescentes, por meio da resolução de problemas e planejamento dos hábitos de vida diária.

No ensaio clínico ${ }^{29}$ referente ao aumento da ingestão de volumes de líquido para manter a saúde do enxerto após o transplante renal, observou-se que a intervenção com uso da garrafa interativa de água por jovens de sete a 19 anos, foi capaz de monitorar a quantidade de líquido ingerida, visando atingir a meta do volume necessário para manter a saúde do rim, calculada de acordo com as características pessoais do paciente. Junto com o uso da garrafa, o grupo de intervenção manteve registro das quantidades de líquido ingeridas diariamente, enquanto o grupo de controle apenas manteve seus registros de acordo com o cuidado usual. Os resultados identificaram maior percentual de ingestão hídrica nos pacientes do grupo de intervenção, com o alcance das metas de ingestão de líquidos comparada ao grupo de controle. Quanto ao uso do gadget, os participantes informaram facilidade de uso, além de trazer conforto com o uso da garrafa.

Estudos menos arrojados identificados nesta revisão 30-31 abordaram intervenções que se utilizaram de estratégias igualmente efetivas. Estudo canadense 30 possibilitou que crianças e jovens transplantados de coração, seus familiares e profissionais da saúde participassem de um acampamento familiar com duração de três dias. Nessa intervenção, foram realizadas atividades recreacionais e educativas, que abordaram temas como fisiopatologia do transplante, fatores de risco, cuidados, tratamento imunossupressor, transição para o cuidado adulto, resolução de problemas e manejo do estresse. Os resultados mostraram que o conhecimento dos participantes e familiares, a respeito de múltiplos aspectos de sua condição de saúde e tratamento aumentou consideravelmente. Além disso, os participantes informaram que o acampamento foi ao encontro de suas expectativas, tendo ocasionado mudanças positivas após a intervenção, tais como maiores níveis de liderança, autoestima, confiança no tratamento proposto.

Finalmente, estudo americano que utilizou vídeos, materiais impressos e instrução verbal para a realização de intervenção educativa, avaliação do conhecimento e das barreiras de receptores pediátricos e cuidadores acerca do uso de proteção solar e estratégias de fotoproteção. ${ }^{31}$ Receptores de órgãos possuem maior risco de desenvolver câncer de pele devido ao uso de imunossupressores, somado à exposição solar sem proteção. Após a realização de estratégias educativas, observou-se melhoria na avaliação do conhecimento de crianças, adolescentes e seus familiares acerca dos fatores de risco para câncer de pele após o transplante. Notou-se ainda, maior frequência no uso do protetor solar, visto que o conhecimento adquirido auxiliou os participantes a entender a importância dos cuidados e dos fatores de risco. Auxiliou também no desenvolvimento de habilidades relacionadas ao autoexame da pele, importante recurso para identificação precoce de alterações que necessitam de avaliação profissional especializada.

Como citado anteriormente, para que o ensino do paciente realizado pelos profissionais de saúde possa demonstrar resultados satisfatórios na recuperação após o transplante, é necessário atentar-se a diversos aspectos que envolvem a forma como as informações são apresentadas ao público alvo e quais estratégias são utilizadas para o ensino das crianças candidatas a e receptoras de órgãos sólidos. Sendo assim, a presente revisão da literatura identificou as seguintes estratégias utilizadas para o ensino no transplante pediátrico: plataformas online e eletrônicas, como Websites e aplicativos móveis; utilização de gadgets que auxiliam na adesão ao tratamento pós-transplante; acampamentos familiares, materiais impressos e vídeo.

O ensino em pediatria é ferramenta eficaz para influenciar a maneira como a doença e seu tratamento são enfrentados, assim, a forma como o ensino é recebido pode gerar diferentes resultados. Para o ensino, podem ser utilizadas variadas técnicas com vistas a mudanças de comportamento, desenvolvimento de habilidades, aconselhamento, fornecimento de informações direcionadas ao paciente, as quais podem ser proporcionadas através de diferentes estratégias. Por meio do ensino, o paciente é capaz de atingir melhorias em sua recuperação, pois a informação contribui sobremaneira para a adesão ao tratamento, melhor estado de saúde e autocuidado. ${ }^{11}$

\section{CONCLUSÃO}

As evidências encontradas nesta revisão identificaram que as estratégias utilizadas para o ensino de candidatos e/ou receptores pediátricos de órgãos sólidos foram exitosas para a melhoria do conhecimento e da qualidade de vida dos pacientes. A maioria dos estudos que compuseram a amostra final tiveram como público alvo 
pacientes adolescentes, sendo fundamental a realização de futuras investigações relacionadas às estratégias de ensino eficazes para público abaixo de 12 anos, por se tratar de estágio de desenvolvimento repleto de necessidades de informação e educação em saúde.

Da mesma maneira, destaca-se a importância de identificar estratégias que possam ser utilizadas nas diversas etapas do tratamento do transplante, quer sejam, pré, intra ou pós-transplante, considerando os benefícios que o ensino pode acarretar, como diminuição do estresse, ansiedade e medo frente ao tratamento futuro, auxiliando na recuperação e no restabelecimento da saúde. Por fim, visto que nos dias atuais crianças e adolescentes estão inseridos na era tecnológica, foi possível identificar que as estratégias educacionais mais utilizadas fizeram uso de plataformas online com uso da internet e de aplicativos móveis, assim como gadgets que facilitaram a adesão ao tratamento.

Este estudo forneceu subsídios sobre a importância do ensino em saúde, bem como identificou a necessidade de novas investigações que abordem estratégias educativas eficazes para o público pediátrico no transplante de órgãos sólidos, visto a ausência de estudos que abordaram essa temática, tanto na literatura brasileira quanto internacional. A produção de novos estudos poderá identificar as estratégias efetivas, contribuindo para a melhoria do bem-estar físico, social e mental do indivíduo e de seus familiares. Os resultados desta revisão integrativa contribuirão para o planejamento de estratégias de ensino-aprendizagem às crianças e adolescentes candidatos e receptores de órgãos.

\section{ABSTRACT}

Purpose: To analyze the evidence available in the literature strategies used to teach pediatric candidates and recipients of solid organ transplantation. Method: An integrative literature review with a selection of articles on the PubMed, EMBASE, CINAHL, and LILACS databases. Results: The use of online and electronic platforms was identified; gadgets to assist in the adherence of the post-transplant treatment; family camps; printed materials and videos as the strategies used in teaching pediatric patient transplantation. Conclusion: There is scarcity of studies both in the national and international literature to teach pediatric patients on transplantation, identifying the need for further investigations, given the importance of the teaching to the continuity of care and better quality of life for pediatric patient.

Keywords: Organ Transplantation; Pediatrics; Teaching Materials; Education; Review.

\section{REFERÊNCIAS}

1. LaRosa C, Baluarte HJ, Meyers KE. Outcomes in pediatric solid-organ transplantation. Pediatric transplantation. 2011;15(2):128-41.

2. Associação Brasileira de Transplante de Órgãos (ABTO). Dimensionamento dos Transplantes no Brasil e em cada estado (2012-2019). Reg Bras Transplantes. 2019; XXV(4):1104.

3. Kamran Hejazi Kenari S, Mirzakhani H, Saidi RF. Pediatric transplantation and tolerance: past, present, and future. Pediatr Transplant. 2014;18(5):435-45.
4. Anton MC, Piccinini CA. O desenvolvimento emocional em crianças submetidas a transplante hepático. Estud Psicol. 2011;16:39-47.

5. Gritti A, Sicca F, Di Sarno AM, Di Cosmo N, Vajro S, Vajro P. Emotional and behavioral problems after pediatric liver transplantation: a quantitative assessment. Pediatr Transplant. 2006;10(2):2059.

6. Yeo M SS. Chronic illness and disability. BMJ. 2005;330:721-3. 
7. Sawyer SM AR. Self-management in adolescents with chronic illness. What does it mean and how can it be achieved? Med J Aust. 2005;183(8):405-9.

8. Shellmer D, Brosig C, Wray J. The start of the transplant journey: referral for pediatric solid organ transplantation. Pediatr Transplant. 2014;18(2):12533.

9. Mendes-Castillo AMC, Bousso RS, Santos MRd, Damião EBC. Estilos de manejo familiar: uma possibilidade de avaliação no transplante hepático pediátrico. Acta Paul Enferm. 2012;25:867-72.

10. Penaforte $\mathrm{KL}$, et al. Transplante cardíaco infantil: perspectivas e sentimentos maternos. Rev Enferm. 2009;13(4):733-40.

11. Golaghaie F, Bastani F. Cross-cultural adaptation of a patient-based tool for evaluating the implementation of patient education in acute care settings. Patient Educ Couns. 2014;96(2):210-5.

12. Mendes KDS, Silveira RCCP, Galvão CM. Revisão integrativa: método de pesquisa para a incorporação de evidências na saúde e na enfermagem. Texto Contexto Enferm. 2008;17(4):758-64.

13. Ouzzani M, Hammady $\mathrm{H}$, Fedorowicz Z, Elmagarmid A. Rayyan-a web and mobile app for systematic reviews. Syst Rev. 2016;5(1):210.

14. Ursi ES, Gavao CM. Prevenção de lesões de pele no perioperatório: revisão integrativa da literatura. Rev Lat Am Enfermagem. 2006;14(1):124-31.

15. Polit DF, Beck CT. Fundamentos de pesquisa em enfermagem. 7a ed. Porto Alegre: Artmed; 2011.

16. Korus M, Stinson JN, Pool R, Williams A, Kagan $S$. Exploring the information needs of adolescents and their parents throughout the kidney transplant continuum. Prog Transplant. 2011;21(1):53-60.

17. Delucchi A, Gutierrez H, Arrellano P, Slater C, Meneses M, Lopez I. Factors that influence nonadherence in immunosuppressant treatment in pediatric transplant recipients: a proposal for an educational strategy. Transplant Proc. 2008;40(9):3241-3.

18. Korus M, Cruchley E, Stinson JN, Gold A, Anthony SJ. Usability testing of the Internet program: "Teens Taking Charge: Managing My Transplant Online". Pediatr Transplant. 2015;19(1):107-17.

19. Tornay E. Educational information for patients and caregivers in paediatric kidney transplant. J Ren Care. 2007;33(3):115-8.
20. Burton JH, Marshall JM, Munro P, Moule W, Snell GI, Westall GP. Rehabilitation and transition after lung transplantation in children. Transplant Proc. 2009;41(1):296-9.

21. Otal D, Wizowski L, Pemberton J, Nagel K, Fitzgerald $\mathrm{P}$, Walton JM. Parent health literacy and satisfaction with plain language education materials in a pediatric surgery outpatient clinic: a pilot study. J Pediatr Surg. 2012;47(5):964-9.

22. McPake D, Burnapp L. Caring for patients after kidney transplantation. Nurs Stand. 2009;23(19):49-57.

23. Adams HA. A perioperative education program for pediatric patients and their parents. AORN J. 2011;93(4):472-81.

24. McLin V, Spahni S, Boggini T, Guardia A, Wildhaber BE, Geissbuhler A. KidsETransplant: a platform for liver-transplanted children. Stud Health Technol Inform. 2013;192:1226.

25. Susi T, Johannesson M, Backlund P. Serious Games : An Overview. Skövde: Institutionen för kommunikation och information; 2007 2007. Report No.: (ISSN) Contract No.: HS- IKI -TR-07-001. Available in: http://urn.kb.se/resolve?ur n=urn:nbn:se:his:diva-1279

26. Wantland JD, Portillo JC, Holzemer LW, Slaughter R, McGhee ME. The Effectiveness of Web-Based vs. NonWeb-Based Interventions: A Meta-Analysis of Behavioral Change Outcomes. J Med Internet Res. 2004;6(4):e40.

27. Shellmer DA, Dew MA, Mazariegos G, DeVito Dabbs A. Development and field testing of Teen Pocket PATH $((R))$, a mobile health application to improve medication adherence in adolescent solid organ recipients. Pediatr Transplant. 2016;20(1):130-40.

28. Foster BJ, Pai ALH, Zelikovsky N, Amaral S, Bell L, Dharnidharka VR, et al. A Randomized Trial of a Multicomponent Intervention to Promote Medication Adherence: The Teen Adherence in Kidney Transplant Effectiveness of Intervention Trial (TAKE-IT). Am J Kidney Dis. 2018; 72(1):30-41.

29. Kullgren KA, Scholl P, Kidwell KM, Hmiel SP. Using an interactive water bottle to target fluid adherence in pediatric kidney transplant recipients: a pilot study. Pediatr Transplant. 2015;19(1):35-41.

30. Nicholas DB, Dodd B, Urschel S, Young A, West LJ. Evaluation of a family camp intervention for children with a heart transplant and their families. Soc Work Health Care. 2016;55(9):752-65.

31. Coughlin CC, Perez M, Kumar MG, Jeffe DB, Bayliss SJ, Sternhell-Blackwell K. Skin cancer risk education in pediatric solid organ transplant patients: an evaluation of knowledge, behavior, and perceptions over time. Pediatr Transplant. 2017;21(2). 